\title{
The Role of the CMB in Redshift Related Departures from the Gao-Solomon Relation
}

\author{
R. Tunnard (iD) and T. R. Greve (i) \\ Department of Physics and Astronomy, University College London, Gower Street, London WC1E 6BT, UK; richard.tunnard.13@ucl.ac.uk \\ Received 2016 June 1; revised 2017 September 3; accepted 2017 September 19; published 2017 October 27
}

\begin{abstract}
A strong correlation between the far-IR and $\mathrm{HCN}(1-0)$ line luminosities, known as the Gao-Solomon relation, has been observed to hold over more than 10 orders of magnitude in the local universe. Departures from this relation at redshifts $\gtrsim 1.5$ have been interpreted as evidence for increased dense gas star formation efficiency in luminous galaxies during the period of peak of star formation in the history of the universe. We examine whether the offsets from the relation can be explained by the hotter Cosmic Microwave Background (CMB) at high redshift, which, due to a loss of contrast against the hotter background, reduces the observable molecular-line flux far more significantly than the far-IR continuum bands. Simple line-of-sight modeling argues for highly significant departures from the Gao-Solomon relation at high redshift for kinetic temperatures $\sim 15 \mathrm{~K}$, while more complex toy-galaxy models based on NGC 1068 suggest a much weaker effect with the galaxy integrated HCN line flux falling by only $10 \%$ at $z=3$, within the intrinsic scatter of the relation. We conclude that, while the CMB is unlikely to explain the deviations reported in the literature, it may introduce a second-order effect on the relation by raising the low-luminosity end of the Gao-Solomon relation in cooler galaxies. A similar examination of the COIR relation finds tantalizing signs of the $\mathrm{CMB}$ having a measurable effect on the integrated $\mathrm{CO}$ emission in highredshift galaxies, but these signs cannot be confirmed with the current data.
\end{abstract}

Key words: galaxies: high-redshift - ISM: molecules - radiative transfer

\section{Introduction}

Studies have found the $\mathrm{HCN}(1-0)$ line emission from the molecular medium in galaxies, which is thought to trace dense molecular gas, to be very well correlated with the IR emission (e.g., Solomon et al. 1992; Gao \& Solomon 2004a, 2004b; Wu et al. 2005, 2010; García-Burillo et al. 2012; Bigiel et al. 2015). This correlation is most often expressed in terms of the canonical Gao-Solomon luminosity relation between IR and $\mathrm{HCN}(1-0)$ line luminosity: $\log L_{\mathrm{IR}}=0.99 \log L_{\mathrm{HCN}}^{\prime}+2.90$ (units of $L_{\odot}$ and $\mathrm{K} \mathrm{km} \mathrm{s}^{-1} \mathrm{pc}^{2}$ ), which was first derived from integrated luminosities of nearby star-forming galaxies, from normal disk galaxies to ultraluminous infrared galaxies (ULIRGs), but subsequently shown to extend, without modification, all the way down to individual star-forming clumps in the Milky Way. This "universality," spanning more than 10 orders of magnitude in luminosity, was interpreted by Wu et al. (2005) as evidence that the HCN emission in starburst galaxies is well described by an optically thin ensemble of Milky Way-like star-forming clumps of dense gas. This interpretation is also consistent with $500 \mathrm{pc}$ resolution observations in the Antennae galaxies (Bigiel et al. 2015). In this scenario, star formation in ULIRGs is fundamentally similar to that in the Milky Way, but with many more star-forming clumps, and therefore also a higher fraction of dense gas, but importantly not a significantly shorter gas-depletion timescale $\left(t_{\text {depl }} \sim M_{\text {dense }} / \mathrm{SFR}\right)$. This interpretation was further supported by analyses of Herschel observations of the $\mathrm{CO}(5-4)$ through to (13-12) transitions of normal star-forming galaxies, which show linear trends with $L_{\mathrm{FIR}}$ over 13 orders of magnitude, with only 0.2-0.3 dex of scatter (Liu et al. 2015; Kamenetzky et al. 2016). Only ULIRGs show deviations from linearity for these high- $J$ transitions, owing to the significant contribution from mechanical heating to the excitation of these lines (see the discussion in Greve et al. 2014).
Extending the $L_{\mathrm{IR}}-L_{\mathrm{HCN}}^{\prime}$ relation to higher redshifts has proven challenging due to the faintness of the $\mathrm{HCN}$ lines (typically $\sim 5-10 \times$ fainter than CO). Both Gao et al. (2007) and Riechers et al. (2007) found that extremely luminous galaxies at high redshift $(z \sim 2.7$ and $z=6.4$ respectively) show a deviation from the Gao-Solomon relation, lying about a factor of 2 above the $z=0$ relation (see Greve et al. 2006). This excess far-IR emission was interpreted as evidence that luminous galaxies at high redshift are forming stars more efficiently than their counterparts in the local universe, even beyond the enhanced star formation efficiency (SFE) found in local (ultra)luminous infrared galaxies by García-Burillo et al. (2012).

A further complication arises from the increasing fraction of active galactic nuclei (AGNs) with redshift (e.g., Eastman et al. 2007). Since AGNs contribute more to the continuum luminosity than to the HCN luminosity, they have the potential to systematically offset galaxies from the Gao-Solomon relation, especially in the most luminous galaxies. This should be contrasted with the potential for AGN activity to induce star formation, and the potential stimulation of $\mathrm{HCN}$ emission via mid-IR pumping as well as chemical-abundance enhancement of HCN by obscured AGNs (Kohno 2005; Lintott \& Viti 2006; Gaibler et al. 2012; Aalto et al. 2015; Imanishi et al. 2016; Izumi et al. 2016).

While it seems that high-redshift galaxies tend to have higher dense gas fractions (as indeed we see in local ULIRGs; Daddi et al. 2010; Tacconi et al. 2010), due to the greater turbulence, there is little and conflicting evidence beyond the offsets from the Gao-Solomon relation to suggest that they have intrinsically higher dense gas star formation efficiencies (e.g., Combes et al. 2013; Magdis et al. 2014).

In this paper, building upon the work of da Cunha et al. (2013) and Tunnard \& Greve (2016), we examine to what extent the hitherto undiscussed effect of the Cosmic Microwave Background $(\mathrm{CMB})$ on the $\mathrm{HCN}(1-0)$ emission can account 
for the observed deviations in the $L_{\mathrm{FIR}}-L_{\mathrm{HCN}}^{\prime}$ relation at high redshifts. Throughout this paper we discuss the line luminosity, ${ }^{1} L_{\mathrm{HCN}(1-0)}^{\prime}$, which we abbreviate to $L_{\mathrm{HCN}}^{\prime}$. For plotting, we adopt cosmological parameters $h=0.75, \Omega_{\mathrm{m}}=0.3$, and $\Omega_{\mathrm{vac}}=0.7$; the values of these parameters have no effect on the conclusions of this work.

\section{Modeling}

We explore the effects of the rising CMB in two ways. First, we examine the effect on single lines of sight. We then explore a more complex model where we set up a toy model of the local active galaxy NGC 1068 and push this model back to higher redshifts. For much of the analysis in this paper, we use the non-LTE molecular line radiative transfer code RADEX (van der Tak et al. 2007) with collisional data from the Leiden Lambda database, specifically HCN collisional rates (Dumouchel et al. 2010) and CO collisional rates (Yang et al. 2010).

In Section 2.3, we repeat the exploration of the effect of the $\mathrm{CMB}$ on the correlation between the $\mathrm{CO}$ line luminosity and the FIR luminosity. In this case, the lower excitation energy and increased optical depth (due to abundance) of the $\mathrm{CO}(1-0)$ line renders it potentially more susceptible to $\mathrm{CMB}$ effects.

\subsection{Theory}

The increasing temperature of the $\mathrm{CMB}$ with redshift can significantly affect both the dust and molecular-line emission from galaxies, especially at $z>2$ (e.g., da Cunha et al. 2013; Narayanan \& Krumholz 2014; Tunnard \& Greve 2016; Zhang et al. 2016). There are two key effects that come into play depending upon the optical thickness of the line. In low opticaldepth regions, the $\mathrm{CMB}$ photons help pump the low- $J$ transitions when the energy of the $\mathrm{CMB}$ photons approaches the excitation energy of the line. In higher optical-depth regions the effect of the CMB on the line emission is negligible; instead there is a significant loss of contrast due to the higher temperature background, which does not propagate through the line-emitting gas due to the high optical depth.

Photons from the background radiation field stimulate the rotational excitation and de-excitation of the molecules, with the greatest effect on the lowest levels, generally leading to an overall increase in the excitation temperatures of the rotational lines. In a low optical-depth environment the escape probability, $\beta$, (the probability for a given point within a cloud that a photon escapes along a given line of sight, or equivalently the probability that a photon from outside the cloud reaches the point) is high. Most of the background radiation field passes through the gas, and emission from the gas adds to the total emission from the cloud and background. In this case, the dominant effect is the CMB's stimulation of the low-level lines. The precise effect is non-trivial to calculate, due to the coupling of the radiation field with the level populations. Nevertheless, insight may be obtained by considering the effect of the background field on the rate equation for the rotational level $i$. Namely, the background introduces the additional term,

$$
\sum_{j \neq i} B_{j i}\left(T_{\mathrm{bg}}\right)\left(B_{j i} n_{j}-B_{i j} n_{i}\right) \beta_{j i}
$$

into the rate equation, where $B_{j i}\left(T_{\mathrm{bg}}\right)$ is the background radiation field with temperature $T_{\mathrm{bg}}$ at the frequency $\nu_{j i}, B_{j i}$ and

$\overline{L_{\mathrm{mol}}^{\prime}=3.25 \times 10^{7}\left(\frac{\nu_{\mathrm{obs}}}{[\mathrm{GHz}]}\right)^{-2}\left(\frac{D_{L}}{[\mathrm{Mpc}]}\right)^{2}(1+z)^{-3}} \frac{\int s_{\mathrm{mol}} \Delta v}{\left[\mathrm{Jy} \mathrm{km} \mathrm{s}{ }^{-1}\right]}$ (Solomon et al. 1992).
$B_{i j}$ are the Einstein coefficients and $\beta_{j i}$ is the escape probability at frequency $\nu_{j i}$. Using a two level system for clarity, this can be expressed as

$$
B_{21}\left(T_{\mathrm{bg}}\right) \beta_{21}\left(e^{-\frac{h \nu_{21}}{k_{\mathrm{B}} T_{\mathrm{ex}}}}-1\right) B_{12} n_{1} .
$$

One can then consider the limiting cases of this term. When molecular excitation is low, the background radiation term becomes negative, as molecules in level 1 are stimulated into level 2 by the background photons. When the molecules are highly excited, the exponential term tends to unity and the effect of the background tends to zero. Notably, the stimulation of the molecules by the background field will increase the excitation temperature: hence the rate equations must be solved iteratively.

When the cloud becomes optically thick the background can no longer be seen through the cloud. In general, the observed intensity is

$$
I_{\nu}=S_{\nu}\left(1-e^{-\tau_{\nu}}\right)+B_{\nu}\left(T_{\mathrm{bg}}\right) e^{-\tau_{\nu}},
$$

where $S_{\nu}$ is the source function, which for the two level molecular rotational system above can be expressed as

$$
S_{\nu_{21}}=\frac{2 h \nu_{21}^{3}}{c^{2}} \frac{1}{e^{\frac{h \nu 21}{k_{\mathrm{B}} T_{\mathrm{ex}}}}-1}=B_{\nu_{21}}\left(T_{\mathrm{ex}}\right)
$$

(e.g., Equation (28) in da Cunha et al. 2013), and for a high optical depth the observed intensity tends to $B_{\nu_{21}}\left(T_{\mathrm{ex}}\right)$, with the background term tending to zero as all of the background field is absorbed by the cloud. In this case, the emission from the cloud at $T_{\mathrm{ex}}$ is seen against a background at $T_{\mathrm{CMB}}$. Therefore, for constant physical conditions, as redshift and $T_{\mathrm{CMB}}$ increase, the contrast $T_{\mathrm{ex}}-T_{\mathrm{CMB}}$ falls, rendering the clouds harder to observe and leading to an underestimate of the true emission if not properly accounted for.

\subsection{Single Lines of Sight: $H C N$}

We investigate whether a hotter CMB can account for the departure from the Gao-Solomon relation seen by Gao et al. (2007) and Riechers et al. (2007). To this end, we take the observed relation at $z=0$ and extrapolate to higher redshifts assuming an average kinetic temperature, $\mathrm{HCN}$ abundance and $\mathrm{H}_{2}$ density. Under the null hypothesis that there is no evolution in the SFE with redshift the inherent HCN and FIR emission will lie on the Gao-Solomon relation, with the redshift related deviation being due to the CMB effects. Therefore, for a given $L_{\mathrm{FIR}}$, we are assuming the same quantity of dense gas, but alter its excitation and observability by increasing the CMB temperature. This leads to an evolution of the Gao-Solomon relation from its local universe form:

$$
\log _{10}\left(L_{\mathrm{FIR}}\right)=0.99 \log _{10}\left(L_{\mathrm{HCN}}^{\prime}\right)+2.90
$$

(Gao \& Solomon 2004b; Gao et al. 2007), to the form that would be observed at redshift $z$ :

$$
\log _{10}\left(L_{\mathrm{FIR}}\right)=0.99 \log _{10}\left(L_{\mathrm{HCN}}^{\prime}(z) \frac{T_{\mathrm{b}}(z=0)}{T_{\mathrm{b}}(z)}\right)+2.90,
$$

where $T_{\mathrm{b}}$ is the line brightness temperature. We plot these adjusted relations in Figure 1 for $z=3$, two different average densities $\left(\bar{n}_{\mathrm{H}_{2}}=1 \times 10^{4} \mathrm{~cm}^{-3}\right.$ and $\left.\bar{n}_{\mathrm{H}_{2}}=1 \times 10^{5} \mathrm{~cm}^{-3}\right)$, an 

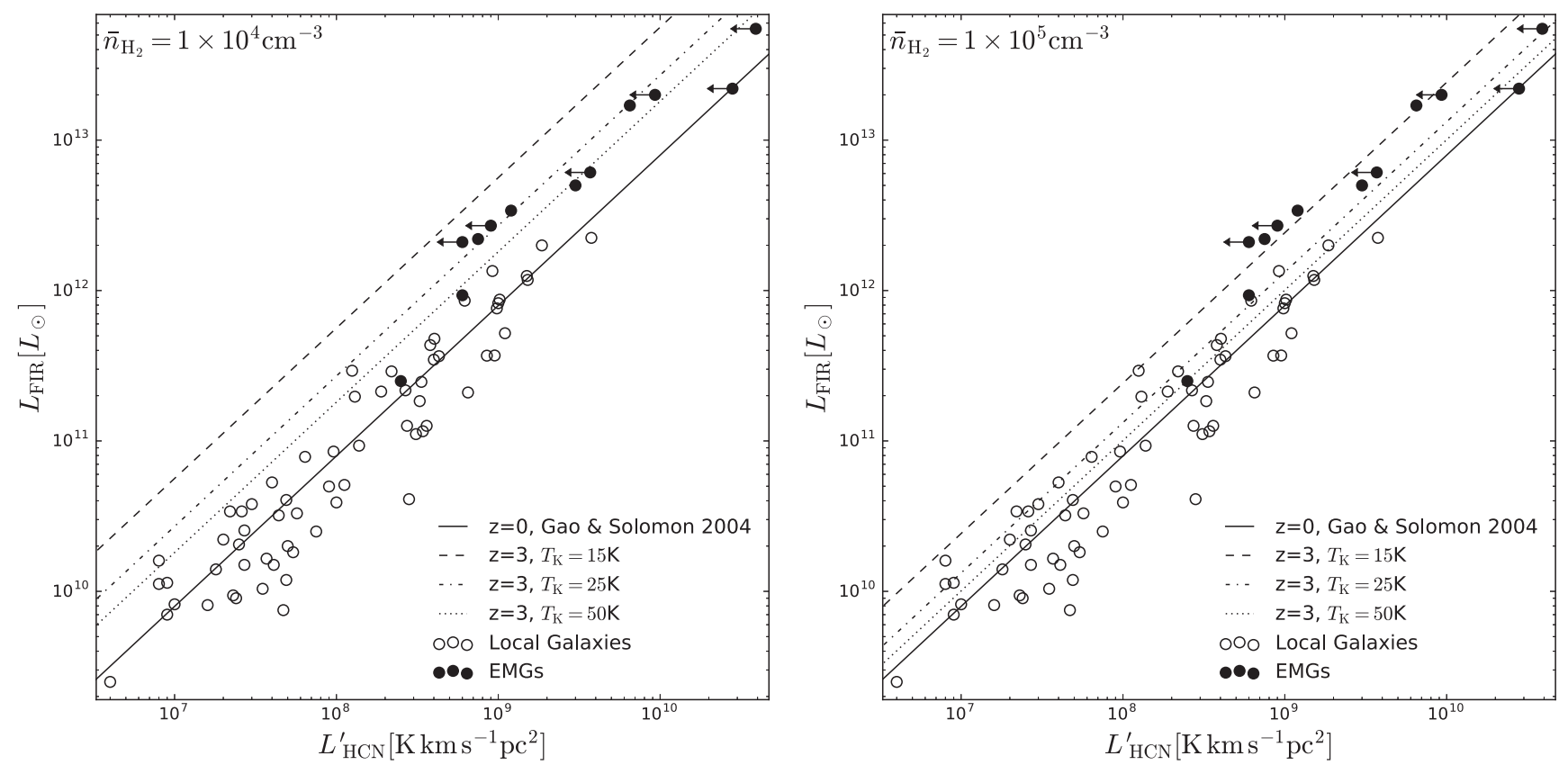

Figure 1. Effect of the CMB on the Gao-Solomon relation at $z=3$ for a range of kinetic temperatures with $\bar{n}_{\mathrm{H}_{2}}=1 \times 10^{4} \mathrm{~cm}^{-3}\left(\right.$ left) and $\bar{n}_{\mathrm{H}_{2}}=1 \times 10^{5} \mathrm{~cm}^{-3}$ (right), calculated using RADEX. Data from Gao et al. (2007) and references therein: Beelen et al. (2006), Carilli et al. (2005), Solomon \& Vanden Bout (2005), Wagg et al. (2005), Egami et al. (2000), Riechers et al. (2006), Isaak et al. (2004), and Greve et al. (2006).

$\mathrm{HCN}$ abundance with respect to $\mathrm{H}_{2}$ of $2 \times 10^{-8}$ and assuming virialized gas $\left(d v / d r=2.7 \mathrm{~km} \mathrm{~s}^{-1} \mathrm{pc}^{-1}\right.$ and $8.5 \mathrm{~km} \mathrm{~s}^{-1} \mathrm{pc}^{-1}$ respectively). These two values of the density were chosen as the critical density of the $\mathrm{HCN}(1-0)$ lines is approximately $1 \times 10^{5} \mathrm{~cm}^{-3}$ (e.g., Greve et al. 2014), but the effective critical density, when taking into account the potential for line trapping, can be as low as $\sim 1 \times 10^{4} \mathrm{~cm}^{-3}$ (Shirley 2015). It should be noted that both of these values are kinetic temperature sensitive but that RADEX was used to calculate the CMB affected brightness temperatures, which calculates the level populations without assuming a critical density.

For local galaxies, Gao \& Solomon (2004b) reported $L_{\mathrm{IR}}$, but in later works (including Gao et al. 2007) it has become conventional to use $L_{\mathrm{FIR}}$. Therefore, the local galaxies used in this paper had to have $L_{\mathrm{FIR}}$ recalculated; for this purpose, we used the IRAS flux definition of $L_{\text {FIR }}$ from Sanders \& Mirabel $(1996)^{2}$ and use the galaxy luminosity distances from Gao \& Solomon (2004b) for consistency and comparability with their $L_{\mathrm{HCN}}^{\prime}$. The $L_{\mathrm{FIR}}$ values for the high-redshift galaxies are taken directly from Gao et al. (2007).

In Figure 2, we plot the $T_{\mathrm{b}, z} / T_{\mathrm{b}, z=0}$ contours as a function of redshift and kinetic temperature for the same parameters as those used in Figure 1. These may be thought of as the generalized correction factor surfaces for the vertical offset in the Gao-Solomon relation due to the two CMB effects discussed above. The contours show the expected rapid fall to zero observability as the kinetic temperature of the gas approaches that of the $\mathrm{CMB}$, with a general trend toward decreased observability with increasing redshift. The lower density surface is much more strongly affected by the CMB as

\footnotetext{
$2 L_{\mathrm{FIR}}=L_{40-500 \mu \mathrm{m}}=4 \pi D_{L}^{2} C\left[1.26 \times 10^{-14}\left\{2.58 f_{60}+f_{100}\right\}\right]$, where $D_{L}$ is the luminosity distance in meters, and $f_{60}$ and $f_{100}$ are the respective IRAS fluxes in jansky. $C$ is a correction factor lying between 1.4 and 1.8; we adopt an average value of 1.6 here as we are not actually fitting to the galaxies.
}

the $\mathrm{HCN}$ in this gas is subthermally excited. Nevertheless, even in the dense case, if the kinetic temperature of the gas is approaching that of the CMB (as it indeed does in cold prestellar GMCs), then the loss of contrast becomes very significant.

Given that we are searching for a shift in observable $\mathrm{HCN}$, we must confirm that there is not also a compensating reduction in $L_{\mathrm{FIR}}$. In Figure 3, we plot the observable fraction of the dust brightness temperature against the $\mathrm{CMB}$ as a function of redshift and observed wavelength for dust temperatures of 20 and $40 \mathrm{~K}$. The surfaces are calculated following the discussion and Equation (18) in da Cunha et al. (2013). The plots in Figure 1 have not been adjusted to include this effect as it depends on the method used to observe and calculate $L_{\mathrm{FIR}}$, which varies for each galaxy. The dust in luminous highredshift galaxies is primarily at temperatures $30-50 \mathrm{~K}$, more closely represented by the $40 \mathrm{~K}$ surface than the $20 \mathrm{~K}$. Nevertheless, even for the $20 \mathrm{~K}$ case, at $z=3$ and an observing wavelength of $1 \mathrm{~mm}$ the $\mathrm{CMB}$ only reduces the observed dust temperature by $10 \%$. For the much more realistic parameters of $40 \mathrm{~K}$ dust and an $850 \mu \mathrm{m}$ measurement the reduction is less than $0.5 \%$, while for the galaxies shown in Figure $1 L_{\mathrm{FIR}}$ was calculated using observations at 60 and $100 \mu \mathrm{m}$, where the effect is less than $0.1 \%$. Therefore, while care should be taken to ensure that the $\mathrm{CMB}$ is not affecting the dust observations of a specific galaxy, it is extremely unlikely to be having any effect on the observations as a whole.

We note that using a naïve analytical approach to the loss of contrast significantly overestimates the effect of the CMB. I.e., if one assumes high optical depth and that:

$$
\begin{aligned}
T_{\mathrm{b}, z} & \simeq T_{\mathrm{b}, z=0}-\left(T_{\mathrm{CMB}, z}-T_{\mathrm{CMB}, z=0}\right) \\
& =T_{\mathrm{b}, z=0}-z T_{\mathrm{CMB}, z=0},
\end{aligned}
$$

then one can significantly underestimate the observable $\mathrm{HCN}$, due to a combination of optical depth effects and the hotter 

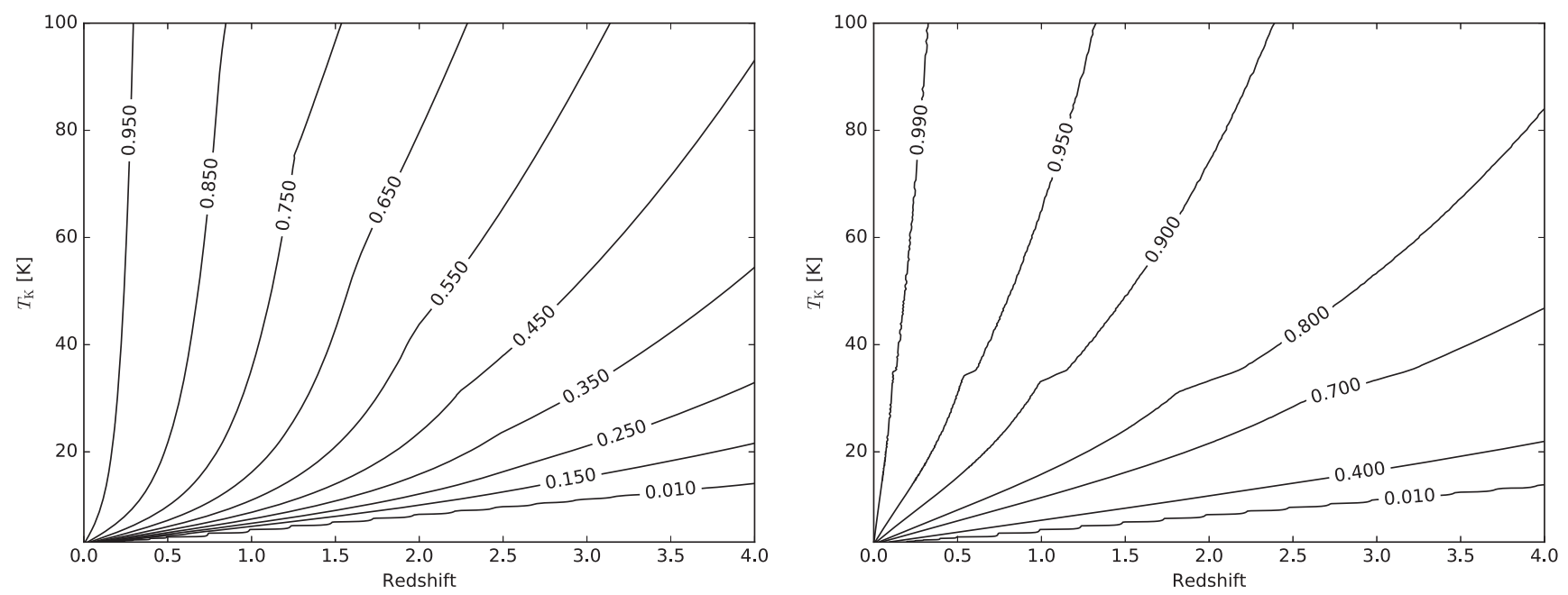

Figure 2. Contour plots of the ratio of the $\mathrm{HCN}(1-0)$ line brightness at the indicated redshift and at $z=0$ for given gas kinetic temperatures for $\bar{n}_{\mathrm{H}_{2}}=1 \times 10^{4} \mathrm{~cm}^{-3}$ (left) and $\bar{n}_{\mathrm{H}_{2}}=1 \times 10^{5} \mathrm{~cm}^{-3}$ (right), calculated using RADEX. Plotting against the kinetic temperature allows us to incorporate the effect of the CMB on the line excitation temperature. The effect of the CMB is far less pronounced in the higher density plot, where the higher collisional excitation rate drives the line excitation temperature closer to the kinetic temperature of the gas. In these plots, the CMB temperature at a given redshift is approximately equal to the 0.01 contour.

CMB contributing to exciting the low- $J$ lines. This is not an issue in the current analysis as RADEX takes the incident background radiation field and its propagation through the gas column into account.

\subsection{Single Lines of Sight: $\mathrm{CO}$}

So far in this paper we have focussed on the effect of the $\mathrm{CMB}$ on the $\mathrm{HCN}(1-0)$ line. However, there is undoubtedly also an effect on the CO lines (e.g., da Cunha et al. 2013). We therefore repeated the previous analysis but for $\mathrm{CO}$, assuming an abundance of $5 \times 10^{-5}$ with respect to $\mathrm{H}_{2}$. We use the galaxies and data from Greve et al. (2014, and references therein), while recalculating $L_{\mathrm{FIR}}$ and $L_{\mathrm{CO}}^{\prime}$ to be consistent with the definitions and cosmology used in this paper. The $40-500 \mu \mathrm{m}$ FIR luminosities of the local (U)LIRGs and highredshift sources were calculated using the CIGALE SED fits performed by Greve et al. (2014). ${ }^{3}$ In Greve et al. (2014), the galaxies were carefully chosen to have well-sampled, and thus well fitted, SEDs.

Fitting to all of the galaxies, using orthogonal distance regression $(\mathrm{ODR})$, we find $\log _{10}\left(L_{\mathrm{FIR}}\right)=(1.04 \pm 0.04) \log _{10}\left(L_{\mathrm{CO}}^{\prime}\right)+$ $(1.5 \pm 0.4)$, consistent with the results of Greve et al. (2014). Note that when fitting only the $z<1$ galaxies with ODR, we find $\log _{10}\left(L_{\mathrm{FIR}}\right)=(0.90 \pm 0.06) \log _{10}\left(L_{\mathrm{CO}}^{\prime}\right)+(2.8 \pm 0.6)$. This relation was then adjusted for redshift in the same manner as for $\mathrm{HCN}$.

The results are shown in Figure 4. We find that the highredshift galaxies are indeed systematically offset from the redshift-zero relation, consistent with a loss of emission from cooler gas at high redshift.

Do high-redshift galaxies really form a separate "population" in the $L_{\mathrm{FIR}}-L_{\mathrm{CO}}^{\prime}$ diagram? With the current data, we can make no conclusive claims, but an examination of the $L_{\mathrm{FIR}} / L_{\mathrm{CO}}^{\prime}$ ratio finds values of $83 \pm 7,95 \pm 12$, and $140 \pm 27$ for $z<1$, $1<z<2.5$, and $z>2.5$ galaxies, respectively. Welch's t-test for the null hypothesis that the local and high-redshift galaxies have the same mean $L_{\mathrm{FIR}} / L_{\mathrm{CO}}^{\prime}$ ratio finds the two to be

\footnotetext{
3 See http://demogas.astro.noa.gr/hercules_seds.htm and http://demogas. astro.noa.gr/DSFGs_seds.htm.
}

inconsistent at the $2.6 \sigma$ level $(p$-value $=0.005){ }^{4}$ Therefore, we find the evidence too weak to claim that the systematic offset of the high-redshift galaxies is real, based on the present data alone. The current analysis also cannot show conclusively that a change in the $L_{\mathrm{FIR}} / L_{\mathrm{CO}}^{\prime}$ ratio is not due to a luminosity dependent effect. Nevertheless, the $1<z<2.5$ and $z>2.5$ galaxies share similar $L_{\mathrm{FIR}}$, suggesting that if the offset is real then the effect is consistent with redshift related effects (including evolution). A confirmed offset could then be due to CMB effects, strengthening the case for either significant reservoirs of cold molecular gas in high-redshift galaxies, which are undetectable with conventional, or for evolution of the CO-IR relation has high redshifts. This is discussed further in Section 3.

\subsection{Galaxy Averaged Effects}

The local active galaxy NGC 1068 has been extensively studied and modeled, most recently by García-Burillo et al. (2014) and Viti et al. (2014). Here, we use the physical conditions from Viti et al. (2014), found by modeling the HCN and $\mathrm{CO}$ line emission, to generate a toy model galaxy which we then shift to higher redshifts. The toy model is based upon that used in Tunnard \& Greve (2016), and uses RADEX to generate an optically thin ensemble ${ }^{5}$ of $10^{4}$ putative clouds across analytically defined temperature and density gradients, with the clouds distributed radially according to an exponential profile with scale length of $1.4 \mathrm{kpc}$. Note that while the ensemble is optically thin there are no such restrictions on the emission from the clouds themselves.

The toy galaxy is separated into two radial regimes: the AGN dominated core out to $r=400 \mathrm{pc}$ followed by the starbursting spiral arms out to $r=1750 \mathrm{pc}$. Since we only

\footnotetext{
4 That is to say, that if the means of the local and high-redshift galaxy populations are in fact the same, and are both normally distributed, then with the sample sizes we have there is a $0.5 \%$ chance of seeing a difference as great or greater than we see here.

5 For example, the clouds are either nonoverlapping or kinematically decoupled. We assume that the unresolved galaxy kinematics lead to a Gaussian profile: we do not model the galaxy kinematics or otherwise include them in the analysis.
} 

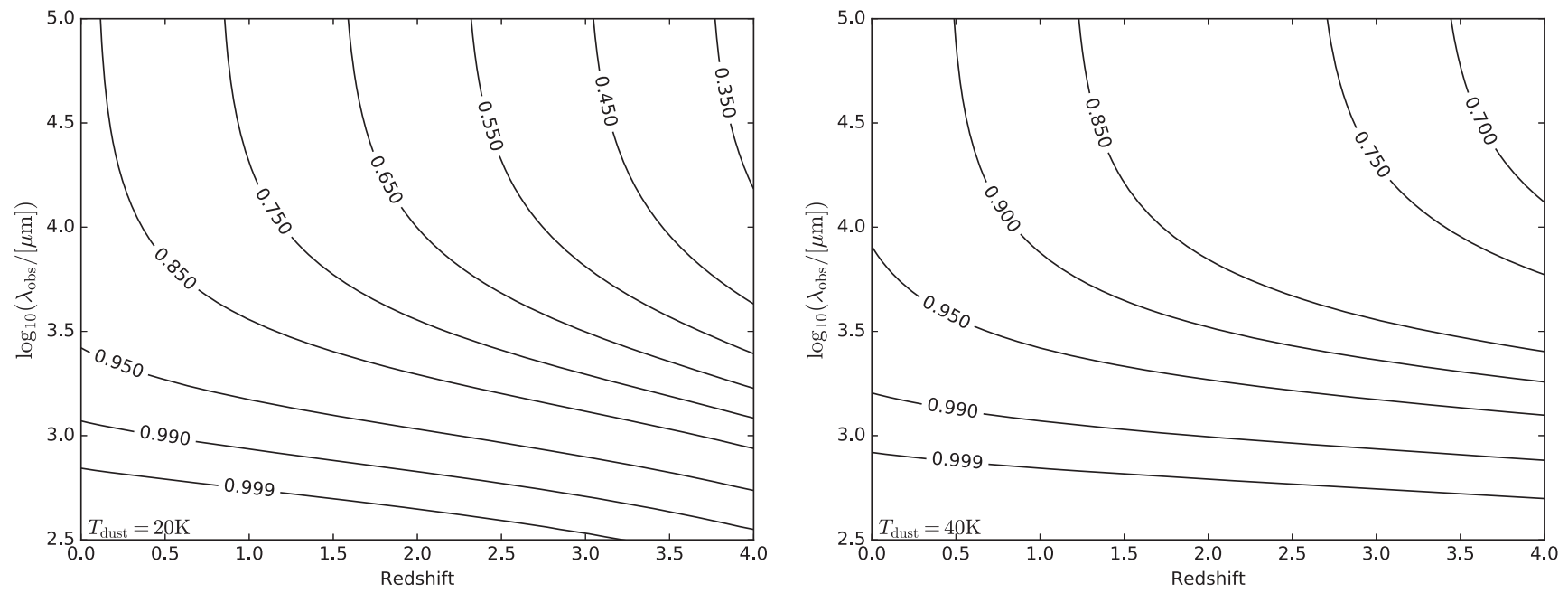

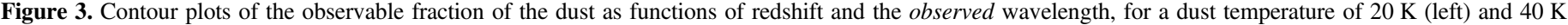

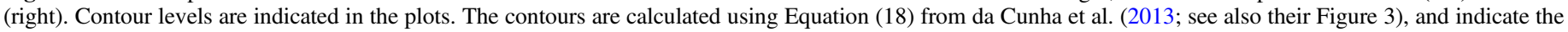
difference between the intrinsic dust brightness temperature and that of the CMB, normalized by that intrinsic dust brightness temperature.

include the effects of the CMB in our model; i.e., we do not consider the effects of cosmological dimming, the radii are largely arbitrary. Over the AGN region, the kinetic temperature falls linearly from 200 to $60 \mathrm{~K}$, and then down to $40 \mathrm{~K}$ at the edge of the starburst. Density falls linearly in log-space, over the same radii as the kinetic temperature, from $10^{6} \mathrm{~cm}^{-3}$ to $10^{5} \mathrm{~cm}^{-3}$ and then $10^{4} \mathrm{~cm}^{-3}$. Throughout we assume $X_{\mathrm{HCN}}=2 \times 10^{-8}$ and virialized gas.

To examine the effect in less active galaxies, we include a cooler version of the model, which is identical but with kinetic temperature pivot points of 100,40 , and $10 \mathrm{~K}$. Note that in no case is the kinetic temperature allowed to fall below the $\mathrm{CMB}$ temperature at the model redshift.

We also run the models with CO. For the CO toy models, we use the same physical parameters as for $\mathrm{HCN}$ (except for $X_{\mathrm{HCN}}=5 \times 10^{-5}$ ), but also add a diffuse $\mathrm{CO}$ field in the form of an additional $10^{4}$ clouds with $n_{\mathrm{H}_{2}}=5 \times 10^{2} \mathrm{~cm}^{-3}$ and $T_{\mathrm{K}}=15 \mathrm{~K}$.

The results of the models are shown in Figure 5, where we plot the integrated galaxy line luminosities, relative to their $z=0$ values, for the various models. We include in Figure 5 three comparison models with constant temperature and density. These models have uniform temperatures of $50 \mathrm{~K}$ and uniform densities of $n_{\mathrm{H}_{2}}=1 \times 10^{4} \mathrm{~cm}^{-3}$ and $n_{\mathrm{H}_{2}}=1 \times$ $10^{5} \mathrm{~cm}^{-3}$ for $\mathrm{HCN}$, and $n_{\mathrm{H}_{2}}=1 \times 10^{3} \mathrm{~cm}^{-3}$ for CO.

Importantly, these toy models allow us to begin to account for variations in the physical parameters across the galaxies, as opposed to assuming a constant temperature and density. This approach avoids the potentially problematic assumption of a constant gas density and temperature across the disk, which can overestimate the effect of the CMB because the hot, dense galactic nuclei are far less affected by the loss of contrast than the cooler outer regions. Unsurprisingly, for $\mathrm{CO}$, the biggest effect is the assumption of a constant temperature, since for reasonable column densities the line is approximately thermalized at densities as low as $1 \times 10^{3} \mathrm{~cm}^{-3}$, whereas HCN is subthermally excited even at $1 \times 10^{4} \mathrm{~cm}^{-3}$. The effect of the hotter central regions of the galaxy, whether they be heated by an AGN, mechanical turbulence (as is the case for the Central Molecular Zone in our own galaxy Ginsburg et al. 2015), or a greater surface density of star formation, is important to consider when trying to predict or interpret high-redshift observations.

\section{Discussion}

We have shown that, to a lesser or greater extent, the loss of contrast against the $\mathrm{CMB}$ with increasing redshift can lead to a shift in the Gao-Solomon relation upwards, increasing the logarithmic intercept. This shift applies whatever the intrinsic trend may be, whether it is super linear, bi-modal or indeed if there is no change in the intrinsic relation (García-Burillo et al. 2012; Usero et al. 2015). These results complements the findings of Lintott \& Viti (2006) who argued that the apparent $\mathrm{HCN}$ deficit in high-redshift galaxies might be due to metallicity evolution; i.e., suggesting that there is no intrinsic evolution in the Gao-Solomon relation with redshift. Nevertheless, the CMB effects do not appear to be significant in galaxies at least as active as NGC 1068, requiring cooler or less dense gas to be prevalent in order to exceed the natural scatter in the relationship.

Interestingly, this could lead to a second-order effect on the Gao-Solomon relation at high redshifts: if more luminous galaxies are, on average, denser and warmer than less luminous galaxies, then as redshift increases there will be a gradual shifting of not only the intercept but also the gradient of the Gao-Solomon relation, i.e., if there is no inherent evolution of the Gao-Solomon relation with redshift then we should observe the gradient decreasing and the intercept increasing due to redshift related effects alone. Unfortunately, this prediction is extremely challenging to test as it requires a statistically significant sampling of less luminous galaxies at high redshift; an observationally unachievable aim at the current time.

The reduced observability of molecular lines at high redshift has further implications, and when considering an entire galaxy there is the additional complication of variations in density and temperature throughout and across a galaxy. Therefore, if a significant fraction of the $\mathrm{HCN}$ emission in a galaxy originates from cool gas near the effective critical density, with a core of much denser gas near the galactic center (reminiscent of the 

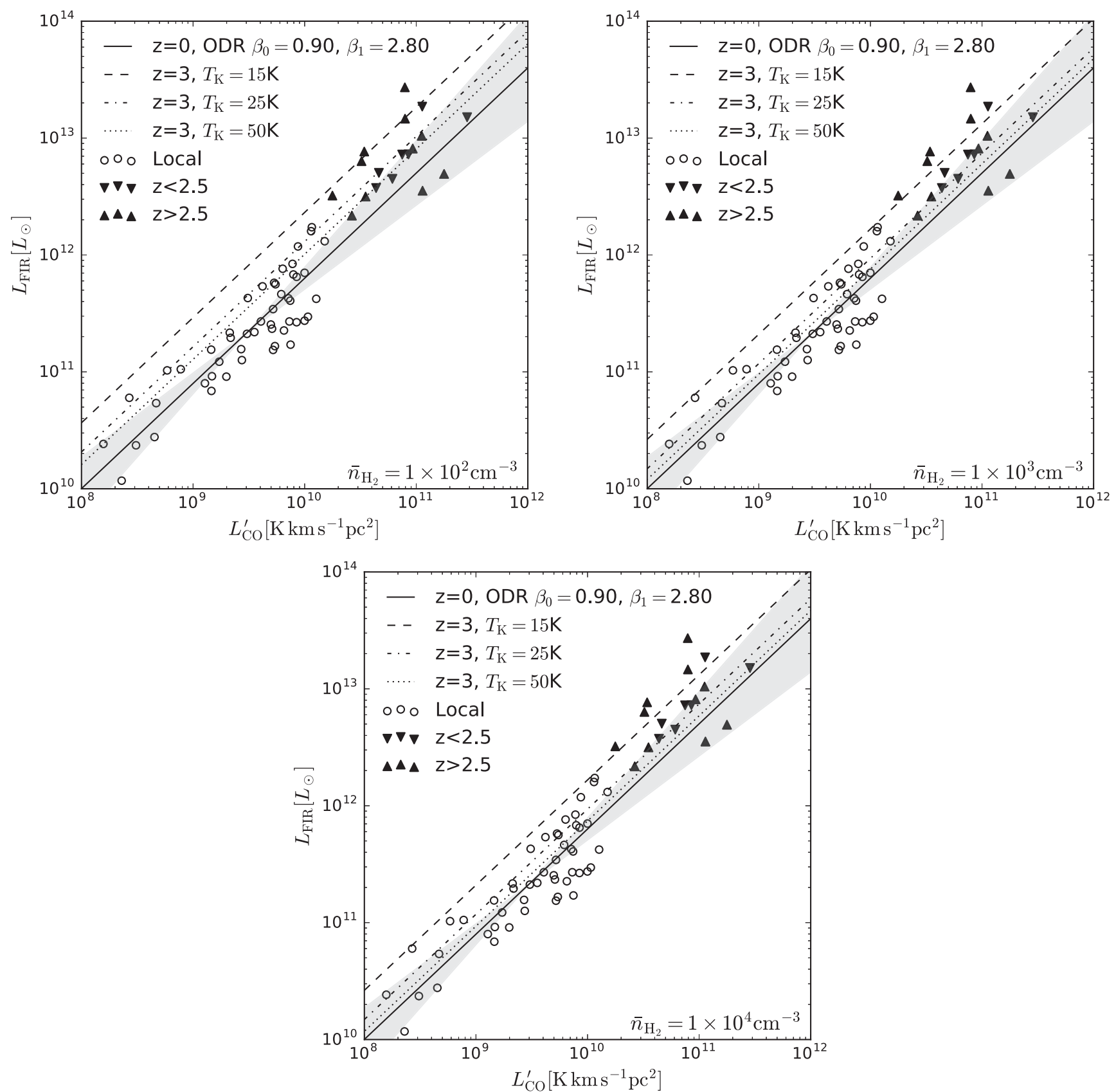

Figure 4. Effect of the CMB on the $L_{\mathrm{FIR}}-L_{\mathrm{CO}}^{\prime}$ relation at $z=3$ for a range of kinetic temperatures with $\bar{n}_{\mathrm{H}_{2}}=1 \times 10^{2} \mathrm{~cm}^{-2}$ (left), $\bar{n}_{\mathrm{H}_{2}}=1 \times 10^{3} \mathrm{~cm}^{-3}$ (right), and $\bar{n}_{\mathrm{H}_{2}}=1 \times 10^{4} \mathrm{~cm}^{-3}$ (bottom) calculated using RADEX. Data are recalculated from Greve et al. (2014) to use $L_{\mathrm{FIR}}$ as defined in the rest of this paper over the range of $40-500 \mu \mathrm{m}$. We fit only the $z<1$ galaxies with orthogonal distance regression (ODR), and find $\log _{10}\left(L_{\mathrm{FIR}}\right)=(0.90 \pm 0.06) \log _{10}\left(L_{\mathrm{CO}}^{\prime}\right)+(2.8 \pm 0.6)$ and is shown by the solid line. The $3 \sigma$ uncertainty in the ODR fit is indicated by the gray-shaded area. The high-redshift galaxies all appear to lie consistently above the $z=0$ relation, consistent with the predicted CMB effects.

Central Molecular Zone in the Milky Way) this can lead to large fractions of the gas being lost against the CMB. Furthermore, if there is a radial density profile across the galaxy, with the average density at the outer edges much lower than the center, this will inevitably bias size measurements with the edges much harder to distinguish from the CMB. This would also likely lead to an artificially high SFE being observed toward the edges of high-redshift galaxies in future resolved studies. The local observations of Gao \& Solomon (2004b), Wu et al. (2010), and Kepley et al. (2014) found that for resolved galaxies the $L_{\mathrm{FIR}} / L_{\mathrm{HCN}}^{\prime}$ ratio increases toward the galactic centers, probably due to increased SFE (Leroy et al. 2013), suggesting that at high redshifts the loss of the outskirts is very possible. This last point is currently controversial with the exact opposite trend seen by Usero et al. (2015) and Bigiel et al. (2016), who found that the $L_{\mathrm{IR}} / L_{\mathrm{HCN}}^{\prime}$ ratio decreases toward the centers of local galaxies. In particular, Bigiel et al. (2016) found evidence suggesting that star formation does not care for the total density but rather the local density contrast.

A more general but related side note is that the increasing CMB will bias molecular gas detections toward hotter gas. This 

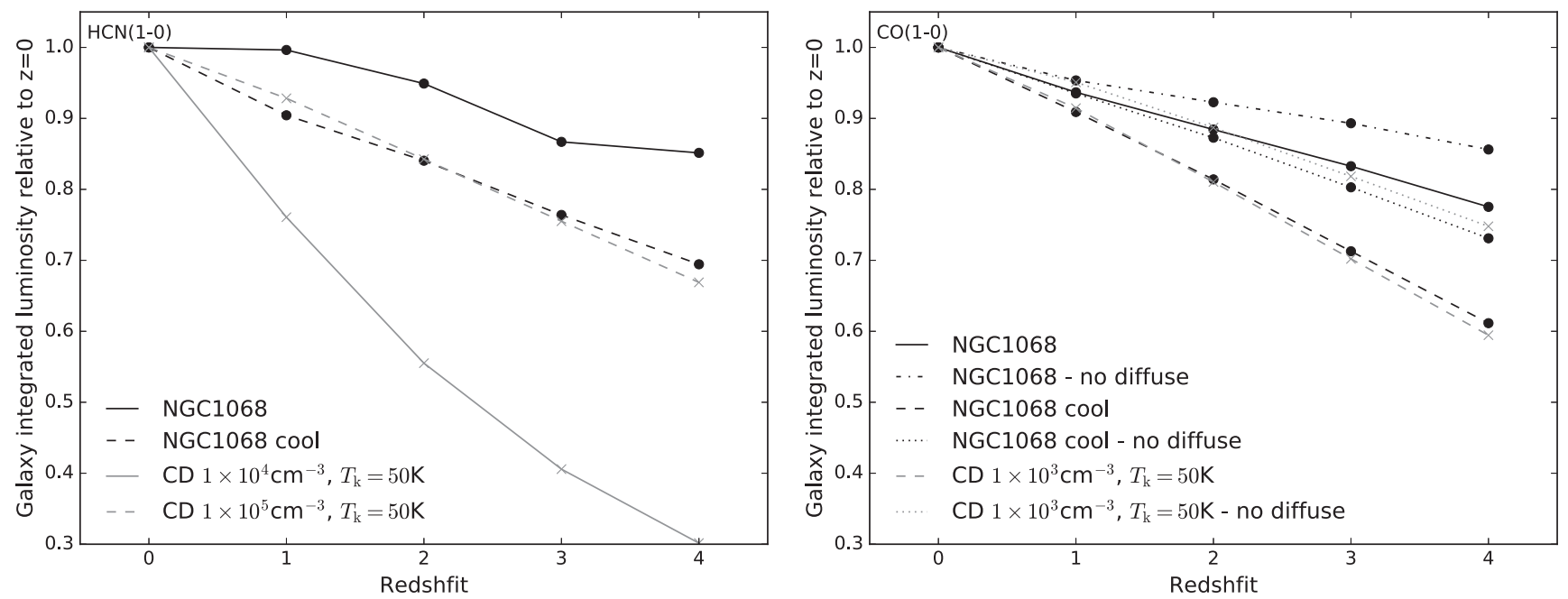

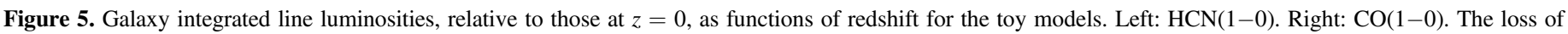
contrast against the CMB is evident even for the NGC 1068 analogue. In both subplots the constant density model (CD) is also plotted in gray with crosses.

is not a new result, but is important to keep in mind in the coming decade as next generation instruments allow for more regular observations of high-redshift molecular lines.

Some "observations" of $\mathrm{HCN}(1-0)$ at high redshift are observations of $\mathrm{HCN}(2-1)$ or higher lines, which are then converted to $\mathrm{HCN}(1-0)$ by making assumptions about the excitation of the gas or using locally calibrated conversions. Not only are these inherently uncertain, but they will be affected by the CMB differently. For the $\operatorname{HCN}(2-1)$ line, we show in the Appendix A that this effect is not significant over the parameter space discussed for the single lines of sight, with the fractional loss in $\mathrm{HCN}(2-1)$ brightness temperature closely following that of $\mathrm{HCN}(1-0)$. The mid to high- $J$ lines, however, are far less significantly affected, although they are troubled by potentially being biased by AGNs, especially the $J=4-3$ line (Riechers et al. 2007; Imanishi et al. 2016; Izumi et al. 2016).

In Section 2.3, we used the data from Greve et al. (2014) to explore the potential effect of the $\mathrm{CMB}$ on the $\mathrm{CO}$ emission in high-redshift galaxies, and found tentative evidence of an offset from the low-redshift relationship, consistent with the predicted CMB effects, but insufficiently significant to make any firm claims. Since the sample was selected to have secure SED fits and $\mathrm{CO}(1-0)$ detections, it is small, with only 20 high-redshift galaxies and 50 local galaxies. While the $L_{\mathrm{FIR}} / L_{\mathrm{CO}}^{\prime}$ ratio does imply that the high-redshift galaxies form a separate population, consistent with CMB effects, the effect is too weak to be confirmed. The offset also depends on the fitting to the local (U)LIRGs: a visual inspection of Figure 4 might suggest that the data would be better fitted if ULIRGs and high-redshift galaxies were fitted together as one population, and the less luminous galaxies fitted as a second. In this case, no redshift related offset would be seen. Physically, this could be motivated by the enhanced dense gas fractions in local ULIRGs and high-redshift galaxies.

Recent, low- $J$, observations of $\mathrm{CO}$ in $17, z>2$, star-forming galaxies by Aravena et al. (2016) show no significant offset of the high-redshift galaxies from local ULIRGs. This calls into question the offset seen in Figure 4, suggesting that it may be merely a sampling effect.

One possibility is that selection effects are leading to galaxies with cooler dust being preferentially identified at high redshift. For local galaxies, $L_{\text {FIR }}$ is calculated using the
IRAS $60 \mu \mathrm{m}$ and $100 \mu \mathrm{m}$ fluxes, while for high-redshift galaxies observations are usually in the region $850-870 \mu \mathrm{m}$ (rest frame $\sim 300 \mu \mathrm{m}$ ). Therefore, for a given bolometric luminosity, there is a selection bias for galaxies with colder dust. However, colder galaxies have a greater fraction of their bolometric luminosity in the far-IR. This might explain why in our Figure 4 we see the high-redshift galaxies as having an excess of far-IR emission, with respect to local (U)LIRGs, whereas Aravena et al. (2016), who plot total IR luminosity ${ }^{6}$ do not see any offset above local ULIRGs. However, the highredshift galaxies in our sample are almost all ULIRGs or even HLIRGs (hyper-luminous infrared galaxies), and as such are expected to have dust as warm as, or even warmer than, the local ULIRGs (e.g., Kovács et al. 2006). It is therefore unclear whether the offset is simply due to random sampling, a systematic bias stemming from dust temperature selection or is a real effect, potentially due to the CMB. Of the three, we consider random sampling to be the most likely culprit.

The toy galaxy models, which attempt to introduce a description of the continuum of physical conditions present within a galaxy, generate contributions from "clouds" with a single density and temperature. The real effect of the CMB may be greater or smaller than predicted here depending upon the temperature, density, and abundance distributions within the observed galaxy. Starburst heated gas and dust is usually cooler and less dense than AGN heated gas (see, for example, the resolved modeling of NGC 1068 in Viti et al. 2014), which means that starburst galaxies are more susceptible to $\mathrm{CMB}$ effects than those dominated by the activity of the central AGN, while normal star-forming galaxies will be the most seriously affected. Furthermore, the presence of an extensive but relatively diffuse $\mathrm{CO}$ reservoir tracing $n_{\mathrm{H}_{2}} \sim 5 \times 10^{2}-10^{3}$ $\mathrm{cm}^{-3}$ will be extremely hard to detect above $z \sim 3$ with the observable flux falling to less than $\sim 0.3$ at $z=3$ and $\sim 0.1$ at $z=4$ (see also Zhang et al. 2016), further biasing attempts to trace molecular gas at these redshifts. Note that the addition of similar diffuse clouds to the HCN toy model has no effect on the integrated emission, as expected given the low column and volume densities. On the other hand, cold and dense gas not yet heated by protostars, not included in the toy models, could

6 Total IR covers the range of $8-1000 \mu \mathrm{m}$. 
contribute significantly to the HCN emission at low redshift: this gas would be much more susceptible to the increased CMB at high redshift.

The toy-galaxy models show that it is important to consider the effect of the CMB on the nonuniform temperatures and densities in galaxies when extrapolating to higher redshift. However, the strength of the effect is extremely sensitive to the kinetic temperature, density, and optical depth of the gas, with many reasonable conditions leading to negligible effects The spatial distribution of the emission is far more sensitive to nonuniformities in the kinetic temperature and density than the galaxy integrated line emission, with the cooler and less dense regions lost much more quickly than the hotter and denser regions. The ideal solution to this problem is a suite of SPH simulations with comprehensive chemical and radiative transfer modeling for which the radiative transfer can be calculated for a range of redshifts.

The CO toy-galaxy models include a diffuse layer of $\mathrm{CO}$ emission spread throughout the disk. While this is reasonable in the local universe, at high redshifts where UV fields may be significantly enhanced with respect to local values the CO may be efficiently photo-dissociated in low density regions. This would exacerbate the loss of $\mathrm{CO}$ emission seen here. Furthermore, the enhanced cosmic-ray rates, which may be found in highly star-forming galaxies due to the increased supernova rate are also capable of effectively dissociating $\mathrm{CO}$, further reducing the $\mathrm{CO}$ emission in the diffuse gas as well as in the denser cores, which are UV shielded but transparent to cosmic rays (Bisbas et al. 2015).

\section{Conclusions}

In this paper, we have described the hitherto unexplored effect of the CMB on the Gao-Solomon relation with a perspective on explaining the apparent offset observed in highredshift galaxies. This effect has been examined both for representative single lines of sight and for toy-galaxy models including a realistic range of physical conditions. While the extent of the effect is strongly sensitive to the temperature and density of the emitting gas there is a predicted systematic offset at $z=3$ for all reasonable physical conditions, although for the warm, dense gas associated with ongoing star formation this effect is small and is much less than the scatter in the GaoSolomon relation.

The increasing significance of the $\mathrm{CMB}$ on the $\mathrm{HCN}(1-0)$ line measurement in cooler galaxies may lead to further variation in the observed Gao-Solomon relation. The cooler, low-luminosity end of the relation may rise (being more strongly affected by the CMB), leading to a sublinear slope. However, if the Gao-Solomon relation is indeed due to galaxy wide $\mathrm{HCN}$ and FIR emission from individual star-forming cores then as the temperature of the cores is largely determined by a (presumably universal) star formation process the kinetic temperature of the emitting $\mathrm{HCN}$ will also be universal, even between low-luminosity normal star-forming galaxies and ULIRGs, and there would be no sublinear slope. On the other hand, if there is substantial mechanical heating of HCN, as in the galactic Central Molecular Zone, the effect may be pronounced.

Extending the examination to $\mathrm{CO}$, we found very tentative signs of a potential offset from the local $L_{\mathrm{FIR}}-L_{\mathrm{CO}}^{\prime}$ relation in high-redshift galaxies, consistent with the predicted CMB effects. However, this offset was found to be too weak to be statistically significant in the present data. Furthermore, the offset is not seen in Aravena et al. (2016), suggesting that the apparent offset in our data may simply be due to random sampling.

While the effect of the CMB is unlikely to be important when extending the Gao-Solomon relation to higher redshifts, owing to it being less than the intrinsic scatter in the relation, the effect may still be important for individual galaxies, especially when resolved. By $z=3$, even the NGC 1068 model lost $10 \%$ of the integrated flux, while the cooler model lost more than 20\%; in both cases, the flux is preferentially lost in the cooler outer regions. This will have significant implications for resolved dense gas studies at $z \gtrsim 2$ unless it is properly accounted for.

Radial temperature and density gradients in galaxies combined with the CMB effects may lead to apparent radial variations in the SFE in high-redshift galaxies, with the suppression of the $\mathrm{HCN}$ line in cooler gas emulating an enhanced SFE in the outer regions of galaxies.

Further study of the effect of the CMB on the observability of galaxy integrated low- $J$ line emission is essential, in particular, a full suite of SPH model galaxies with chemical modeling and radiative transfer would allow for a meaningful exploration of the systematic effects of the CMB on unresolved $\mathrm{HCN}(1-0)$ observations. The assumption of single "average" densities and temperatures to represent a galaxy can have significant effects on conclusions compared to more realistic ranges of parameters.

This study demonstrates that a single unresolved line is insufficient when observing at high redshift, as the additional affect of the CMB enormously complicates the interpretation. With the $\mathrm{HCN}(1-0)$ line alone it is impossible to distinguish between a compact, hot galaxy where all of the dense molecular gas is observed and an extended, cooler galaxy with significant loss of observable emission against the hotter CMB, a problem not found in the local universe or at other wavelengths.

This research is supported by an STFC PhD studentship. T.R.G. acknowledges support from an STFC Advanced Fellowship. We thank the anonymous referee for their suggestions, which improved the paper. This research has made use of the NASA/ IPAC Extragalactic Database (NED), which is operated by the Jet Propulsion Laboratory, California Institute of Technology, under contract with the National Aeronautics and Space Administration.

\section{Appendix A Sensitivity to line transition}

As was discussed in the main text, due to difficulties in observing the highly redshifted $\mathrm{HCN}(1-0)$ line it is often estimated from observations of higher transitions before converting this (with implicit assumptions regarding excitation) to the 1-0 line. While we do not explore the effects of the assumptions here, we do assess how much weaker the effect of the CMB on the $2-1$ line is. The results are shown in Figure 6. For the 2-1 line, there is no significant difference over the parameter range explored for the single lines of sight. However, for the galaxy integrated emission, the effects become much more complicated due to partial stimulation of the line by the CMB and excitation gradients across the galaxy. Nevertheless, the line tends to be similarly affected (Tunnard \& Greve 2016), 

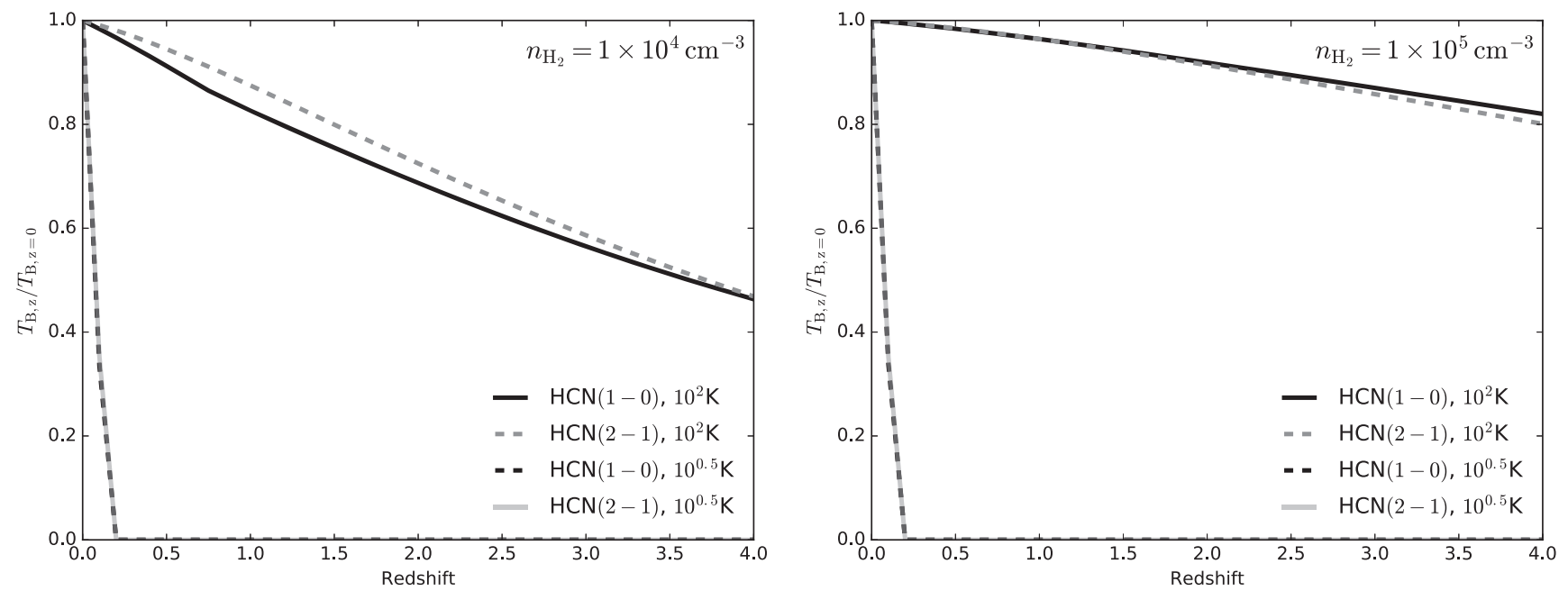

Figure 6. Demonstration that the loss of contrast against a hot CMB is not much alleviated for single lines of sight by using the HCN $(2-1)$ line. For the parameters discussed in Section 2.2 the $\mathrm{HCN}(2-1)$ line is affected almost identically to the $\mathrm{HCN}(1-0)$ line. Results shown here for the hot and cold limits of $10^{2} \mathrm{~K}$ and $10^{0.5} \mathrm{~K}$ on the earlier models for $\bar{n}_{\mathrm{H}_{2}}=1 \times 10^{4} \mathrm{~cm}^{-3}$ (left) and $\bar{n}_{\mathrm{H}_{2}}=1 \times 10^{5} \mathrm{~cm}^{-3}$ (right).

and the stimulated emission is a small effect (Shirley 2015). Higher $J$ transitions will be much less affected by the CMB due to the rapid fall off of the Blackbody spectrum with decreasing wavelength, although attempts to use these lines as proxies for the $J=1-0$ line are plagued by uncertainties in the excitation of the gas, which render them extremely uncertain.

\section{Appendix B HCN-CO Ratios}

Secondary to the discussion at hand but nevertheless interesting is the question of whether and in what way the CMB affects the $L_{\mathrm{HCN}}^{\prime} / L_{\mathrm{CO}}^{\prime}$ ratio. This is harder to model without full galaxy simulations due to the increased range of possible parameters, and we do not investigate all possibilities here. However, we plot in Figure 7 a selected sample of conditions at $z=3$, adjusting the empirical relation found by Gao \& Solomon (2004b).

This very simple exploration does not include temperature effects, in particular, different temperatures for the two species. Furthermore, it does not take into account the structure and density gradients present in real galaxies, which will introduce additional, potentially significant, offsets. For example, see Figure 6 in Viti et al. (2014), where the $\mathrm{HCN}(1-0) / \mathrm{CO}(1-0)$ ratio varies by a factor of 10 across NGC 1068 . This makes the problem of extrapolating to high redshifts far more complicated than for a single species alone and requires careful modeling of a galaxy to achieve reliable results.

\section{ORCID iDs}

R. Tunnard (i) https://orcid.org/0000-0002-7558-7446

T. R. Greve (1) https://orcid.org/0000-0002-2554-1837

\section{References}

Aalto, S., Martín, S., Costagliola, F., et al. 2015, A\&A, 584, A42 Aravena, M., Spilker, J. S., Bethermin, M., et al. 2016, MNRAS, 457, 4406 Beelen, A., Cox, P., Benford, D. J., et al. 2006, ApJ, 642, 694

Bigiel, F., Leroy, A. K., Blitz, L., et al. 2015, ApJ, 815, 103

Bigiel, F., Leroy, A. K., Jiménez-Donaire, M. J., et al. 2016, ApJL, 822, L26

Bisbas, T. G., Papadopoulos, P. P., \& Viti, S. 2015, ApJ, 803, 37

Carilli, C. L., Solomon, P., Vanden Bout, P., et al. 2005, ApJ, 618, 586

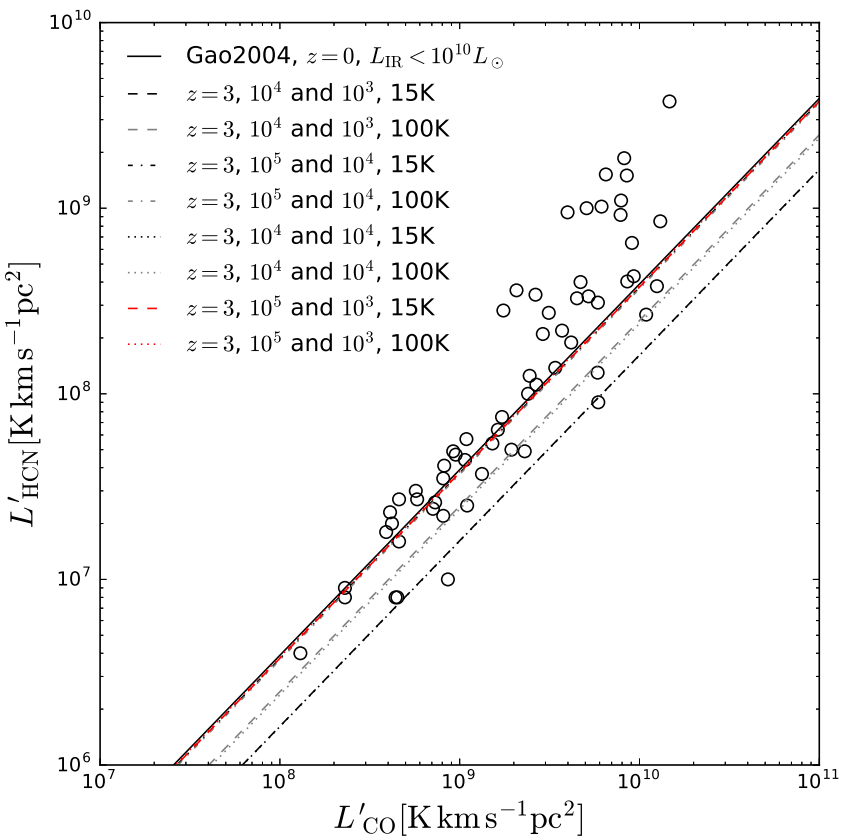

Figure 7. Potential effect of the $\mathrm{CMB}$ on the $L_{\mathrm{HCN}}^{\prime} / L_{\mathrm{CO}}^{\prime}$ ratio, a proxy for the dense gas fraction, for a select sample of parameters. The effect is negligible for most parameters, with the greatest effect seen when HCN is least affected and contrary to the data. Different temperatures, densities, and indeed galaxy integrated measurements will affect this plot, but it is hard to find a realistic set of conditions that could lead to observations overestimating the dense gas fraction. The excess of $\mathrm{HCN}$ in the high luminosity galaxies may indeed be due to a real excess of dense gas.

Combes, F., García-Burillo, S., Braine, J., et al. 2013, in IAU Symp. 292, Molecular Gas, Dust, and Star Formation in Galaxies, ed. T. Wong \& J. Ott (Cambridge: Cambridge Univ. Press), 303

da Cunha, E., Groves, B., Walter, F., et al. 2013, ApJ, 766, 13

Daddi, E., Bournaud, F., Walter, F., et al. 2010, ApJ, 713, 686

Dumouchel, F., Faure, A., \& Lique, F. 2010, MNRAS, 406, 2488

Eastman, J., Martini, P., Sivakoff, G., et al. 2007, ApJL, 664, L9

Egami, E., Neugebauer, G., Soifer, B. T., et al. 2000, ApJ, 535, 561

Gaibler, V., Khochfar, S., Krause, M., \& Silk, J. 2012, MNRAS, 425, 438

Gao, Y., Carilli, C. L., Solomon, P. M., \& Vanden Bout, P. A. 2007, ApJL, 660, L93

Gao, Y., \& Solomon, P. M. 2004a, ApJS, 152, 63

Gao, Y., \& Solomon, P. M. 2004b, ApJ, 606, 271

García-Burillo, S., Combes, F., Usero, A., et al. 2014, A\&A, 567, A125 
García-Burillo, S., Usero, A., Alonso-Herrero, A., et al. 2012, A\&A, 539, A8

Ginsburg, A., Walsh, A., Henkel, C., et al. 2015, A\&A, 584, L7

Greve, T. R., Hainline, L. J., Blain, A. W., et al. 2006, AJ, 132, 1938

Greve, T. R., Leonidaki, I., Xilouris, E. M., et al. 2014, ApJ, 794, 142

Imanishi, M., Nakanishi, K., \& Izumi, T. 2016, ApJ, 825, 44

Isaak, K. G., Chandler, C. J., \& Carilli, C. L. 2004, MNRAS, 348, 1035

Izumi, T., Kohno, K., Aalto, S., et al. 2016, ApJ, 818, 42

Kamenetzky, J., Rangwala, N., Glenn, J., Maloney, P. R., \& Conley, A. 2016, ApJ, 829, 93

Kepley, A. A., Leroy, A. K., Frayer, D., et al. 2014, ApJL, 780, L13

Kohno, K. 2005, in AIP Conf. Ser. 783, The Evolution of Starbursts, ed. S. Hüttmeister et al. (Melville, NY: AIP), 203

Kovács, A., Chapman, S. C., Dowell, C. D., et al. 2006, ApJ, 650, 592

Leroy, A. K., Walter, F., Sandstrom, K., et al. 2013, AJ, 146, 19

Lintott, C., \& Viti, S. 2006, ApJL, 646, L37

Liu, D., Gao, Y., Isaak, K., et al. 2015, ApJL, 810, L14

Magdis, G. E., Rigopoulou, D., Hopwood, R., et al. 2014, ApJ, 796, 63

Narayanan, D., \& Krumholz, M. R. 2014, MNRAS, 442, 1411

Riechers, D. A., Walter, F., Carilli, C. L., et al. 2006, ApJ, 650, 604
Riechers, D. A., Walter, F., Carilli, C. L., \& Bertoldi, F. 2007, ApJL, 671, L13

Sanders, D. B., \& Mirabel, I. F. 1996, ARA\&A, 34, 749

Shirley, Y. L. 2015, PASP, 127, 299

Solomon, P. M., Downes, D., \& Radford, S. J. E. 1992, ApJL, 398, L29

Solomon, P. M., \& Vanden Bout, P. A. 2005, ARA\&A, 43, 677

Tacconi, L. J., Genzel, R., Neri, R., et al. 2010, Natur, 463, 781

Tunnard, R., \& Greve, T. R. 2016, ApJ, 819, 161

Usero, A., Leroy, A. K., Walter, F., et al. 2015, AJ, 150, 115

van der Tak, F. F. S., Black, J. H., Schöier, F. L., Jansen, D. J., \& van Dishoeck, E. F. 2007, A\&A, 468, 627

Viti, S., García-Burillo, S., Fuente, A., et al. 2014, A\&A, 570, A28

Wagg, J., Wilner, D. J., Neri, R., Downes, D., \& Wiklind, T. 2005, ApJL, 634, L13

Wu, J., Evans, N. J., II, Gao, Y., et al. 2005, ApJL, 635, L173

Wu, J., Evans, N. J., II, Shirley, Y. L., \& Knez, C. 2010, ApJS, 188, 313

Yang, B., Stancil, P. C., Balakrishnan, N., \& Forrey, R. C. 2010, ApJ, 718, 1062

Zhang, Z.-Y., Papadopoulos, P. P., Ivison, R. J., et al. 2016, RSOS, 3, 160025 